\title{
Cine dyscontractility index: a novel marker of mechanical dyssynchrony that predicts response to cardiac resynchronisation therapy
}

Konrad Werys ${ }^{1,2}$, MS; Joanna Petryka-Mazurkiewicz ${ }^{2}, \mathrm{MD}, \mathrm{PhD}$; Lukasz Błaszczyk ${ }^{1}$, MS; Jolanta Miśko², MD, PhD; Mateusz Śpiewak ${ }^{2}, \mathrm{MD}, \mathrm{PhD}$; Łukasz A. Małek², MD, PhD Łukasz Mazurkiewicz ${ }^{2}, \mathrm{MD}, \mathrm{PhD}$; Barbara Miłosz-Wieczorek², MD; Magdalena Marczak ${ }^{2}$ MD, PhD; Agata Kubik ${ }^{1,2}$, MS; Agnieszka Dąbrowska ${ }^{2}$; Ewa Piątkowska-Janko ${ }^{1}, \mathrm{PhD}$; Błażej Sawionek ${ }^{1}$, PhD; Rohan Wijesurendra ${ }^{3}$, MD; Stefan K. Piechnik ${ }^{3}$, PhD; Piotr Bogorodzki ${ }^{1}, \mathrm{PhD}$;

1. Institute of Radioelectronics and Multimedia Technology, Warsaw University of Technology, Nowowiejska15/19, 00-665 Warsaw, Poland.

2. Cardiac Magnetic Resonance Unit, Institute of Cardiology, Alpejska 42,04-628 Warszawa, Poland.

3. Division of Cardiovascular Medicine, Oxford Centre for Clinical Magnetic Resonance Research, University of Oxford, Oxford, UK.

\section{CORRESPONDING AUTHOR:}

Konrad Werys

Institute of Radioelectronics and Multimedia Technology, Warsaw University of Technology ul. Nowowiejska15/19, 00-665 Warsaw, Poland

tel: +48791135998

fax: +48228255248

konradwerys@gmail.com 


\section{GRANT SUPPORT}

The study is supported by the Ministry of Science and Higher Education of the Republic of Poland (“Iuventus Plus"' programme, project number: 0477/IP1/2015/73).

K. Werys is supported by National Science Center, Poland (stipend 2014/12/T/ST7/00670).

S. Piechnik is supported by the National Institute for Health Research (NIHR) Oxford Biomedical Research Centre based at The Oxford University Hospitals Trust at the University of Oxford

R. Wijesurendra acknowledges support from the BHF Centre of Research Excellence, Oxford (RE/08/004).

\section{RUNNING TITLE}

Cine dyscontractility index 


\begin{abstract}
Purpose To investigate whether MRI cine-derived dyssynchrony indices provide additional information compared to conventional tagged MRI (tMRI) acquisitions in heart failure patients undergoing cardiac resynchronisation therapy (CRT).

Materials and Methods Patients scheduled for CRT $(n=52)$ underwent pre-procedure MRI including cine and tMRI acquisitions. Segmental strain curves were calculated for both cine and tMRI to produce a range of standard indices for direct comparison between modalities. We also proposed and evaluated a novel index of "dyscontractility", which detects the presence of focal areas with paradoxically positive circumferential strain.

Results Across conventional strain indices, there was only moderate-to-poor $(\mathrm{R}=0.3-0.6)$ correlation between modalities; eight cine-derived indices showed statistically significant $(p<0.05)$ relations to CRT outcome compared to just two tMRI-based counterparts. The novel dyscontractility index calculated on basal slice cine images (cine dyscontractility index, "CDI") was the single best predictor of clinical response to $\mathrm{CRT}(\mathrm{AUC}=0.81, \mathrm{p}<0.001)$. While poorly correlated to its tMRI counterpart $(\mathrm{R}=0.33)$, CDI performed significantly better in predicting response to CRT $(\mathrm{p}<0.005)$, and was also numerically better than all other tMRI indices (AUC 0.53 - 0.76, all $\mathrm{p}$ for AUC comparisons $<0.17$ ).
\end{abstract}

Conclusion Cine-derived strain indices offer potentially new information compared to tMRI. Specifically, the novel cine dyscontractility index (CDI) is most strongly linked to response to cardiac resynchronization therapy in a contemporary patient cohort. It utilizes readily available MRI data, is relatively straightforward to process, and compares favourably with any conventional tagging index.

Keywords Cardiac Magnetic Resonance Imaging, cine, Image Registration, Strain, Cardiac Resynchronization Therapy, Dyssynchrony Index. 


\section{BACKGROUND}

Heart failure is a major public health issue, affecting over 23 million patients worldwide and carrying a substantial risk of morbidity and premature mortality (1). In a large proportion of heart failure patients, dyssynchrony of ventricular contraction (often due to bundle branch block) can induce regional loading disparities and reduce the overall efficiency of ventricular pump function (2). Cardiac resynchronization therapy (CRT) is recommended in selected patients with heart failure, systolic dysfunction and prolonged QRS interval, and has been shown to reduce mortality, symptoms and hospitalizations (3). However, one major limitation of CRT is that up to $30-50 \%$ of patients are "non-responders", who do not experience any meaningful clinical improvement following CRT implantation $(4,5)$. Hence, there is an increasing need for imaging techniques that can reliably quantify pre-implantation mechanical dyssynchrony, improve patient selection, and reduce the incidence of non-response.

While echocardiographic measures of dyssynchrony have only modest sensitivity and specificity for CRT response prediction in multi-center studies (5), progress in cardiac magnetic resonance methods suggests that this modality may prove a more reliable predictor (6-8). Dedicated MR sequences for quantitative assessment of motion include tagged MRI (tMRI) and Displacement Encoding with Stimulated Echoes (DENSE). While the strain indices derived by these methods have been used in the research setting, they have not gained wider clinical popularity. This may reflect the need for acquisition of dedicated MR sequences, and the time-consuming post-processing required. Therefore, application of unsupervised image analysis techniques to ubiquitous cine MRI data could promote the wider clinical application of strain analysis (9). 
In this study, we aimed to develop an unsupervised method for calculation of complete displacement maps directly from cine MRI and then compare indices derived from these data to established tMRI dyssynchrony indices in the ability to predict CRT response in patients.

\section{MATERIALS AND METHODS}

Ethical approval was granted for all study procedures and all subjects gave written informed consent.

\section{Study Population}

A cohort of patients scheduled for CRT $(n=52)$ who underwent MR prior to the procedure was included. Inclusion criteria were: NYHA class II-IV despite optimal medical treatment for at least 3 months, $\mathrm{LVEF}<35 \%$ and QRS $>120 \mathrm{~ms}$. Exclusion criteria and details of study procedures have been published previously (7). Baseline characteristics of the cohort are presented in Table 1.

After 6 months follow-up, patients were classified as responders $(n=26)$ or non-responders $(n=26)$; response was defined as:

- No hospital admission for decompensated heart failure prior to 6-month follow-up, and

- Improvement in NYHA functional class by at least one point or $10 \%$ increase in peak oxygen consumption measured with cardio-pulmonary exercise test as compared with baseline.

\section{MRI Acquisition}

All data were collected on a 1.5 Tesla clinical MR system (Magnetom Avanto, Siemens Healthcare, Erlangen, Germany) with body matrix surface coil.

Cardiac cine bSSFP (balanced steady state free precession) sequence was acquired using retrospective cardiac gating. Typically 25 phases were acquired in 2-, 3- and 4- chamber long axis views and for a stack of short axis views. Scan parameters: echo time $1.2 \mathrm{~ms}$, effective 
repetition time $33-54 m s$, echo spacing $2.7 \mathrm{~ms}$, flip angle $64-79^{\circ}$, slice thickness $8 \mathrm{~mm}$, gap $1.6 \mathrm{~mm}$, in plane image resolution $1.6 \times 1.6 \mathrm{~mm}$.

Tagged images were acquired with a prospectively gated Flash sequence with spatial modulation of magnetization and view-sharing in basal, mid and apical short axis views (one slice each) and also in 2- and 4-chamber long axis views. Typically 18 phases were acquired using scan parameters: tag spacing $6 \mathrm{~mm}$, echo time TE $3.85 \mathrm{~ms}$, effective repetition time TR $40 \mathrm{~ms}$, echo spacing $4.5 \mathrm{~ms}$, flip angle $10^{\circ}$, slice thickness $7 \mathrm{~mm}$, in plane resolution $1.4 \times 1.4 \mathrm{~mm}$.

\section{Cine Image Analysis}

Cine image analysis was performed with dedicated software written in Matlab (Mathworks, USA) and made available as freeware ${ }^{1}$.

\section{Strain Calculation}

The cine strain calculation algorithm was based on widely used unsupervised non-rigid 2D image registration methods, as described previously (10). Briefly, the displacements between consecutive images in time series were calculated using an algorithm similar to the one described by Rueckert et al. (11). The similarity measure was calculated for the whole image, so features like papillary muscles, trabeculae and right ventricle insertion points were by definition used, without being extracted. In order to reduce the calculation time to acceptable levels, in the cost function we used direct similarity measure (squared differences between pixel intensities) regularized by a transformation smoothness measure (weight 0.001). The displacement fields were calculated separately forward and backward between consecutive images and combined together as described by Ledesma-Carbayo et al. (12). Eulerian strain tensor was then calculated; representative maps of the circumferential component of strain tensor are presented (Figure 1; right panels).

\footnotetext{
${ }^{1}$ http://mrkonrad.github.io/MRKonrad/MRViewer
} 


\section{Image Segmentation}

Using the calculation described above, the displacement and strain waveforms could be calculated for every pixel or region in any cine frame, mapping the motion between the frames. We chose to segment the end-diastolic image manually with endocardial and epicardial contours as the point of reference in this study. The myocardial ring was further divided into 6 segments from the indicated right ventricular insertion point. This allowed calculation of segmental median strain in a single frame of reference; "strain plots" (Figure 1; left panels) could be derived and used to calculate dyssynchrony indices.

Short axis slices were classified as basal, mid-cavity or apical $(35 \%, 35 \%$ and $30 \%$ of slices including left ventricle muscle) according to American Heart Association guidelines (13). The first basal slice was defined as the most basal slice where no left ventricular outflow tract was visible at end-systole. The last apical slice was defined as the most apical slice with residual blood visible in the left ventricular cavity at end-systole.

\section{Tagged MRI Images Analysis}

For comparison of performance, tagged MRI (tMRI) strain analysis was performed using SinMod (14), as described previously (7) by two operators (JPM, KW) with 8 years and 5 years of experience respectively.

\section{Calculation Of Traditional Dyssynchrony Indices}

We limited our analysis to circumferential strain because cardiac muscle fibres have a primarily circumferential orientation (15), and the dynamic range of circumferential strain is higher than for other directions of strain (16). We present several established traditional dyssynchrony markers each calculated on strain plots separately obtained from cine and tMRI.

The traditional measures of dyssynchrony focus on absolute timing differences to peak strain between different myocardial segments. The variability of the timing differences (Strain 
Dyssynchrony Index $E_{c c}{ }^{S D I}$ ) is commonly used in echocardiography studies and has also been used in MRI (17) (Figure 2A).

Helm et al. (16) proposed a measure based on visualising the motion as vectors arising from the idealised circular myocardial outline that are scaled by the respective strain values (Figure 2B). In fully symmetric motion, those vectors balance out and their sum (regional variance vector $R V V$ ) remains close to zero for the whole heart cycle. We use the maximum observed value as the individual summary value of dyssynchrony $\left(E_{c c}{ }^{M a x R V V}\right.$ in Figure 2B).

An obvious appearance of dyssynchrony is fanning out of the strain curves as they progress along the time direction. This can be formally assessed by calculating the inter-segmental strain variance for each frame, as also proposed by Helm et al. (16), and then summarised as the maximum for each data set $\left(E_{c c}{ }^{\text {MaxVar }}\right.$ in Figure $\left.2 C\right)$.

For completeness, we also assessed $E_{c c}{ }^{C U R E}$ (Circumferential Uniformity Ratio Estimate), which is also known as US (Uniformity of Strain) $(6,8,16-20)$. This is based on advanced Fourier analysis of the harmonics in segmental strains to reveal the motion symmetry. Unlike the other indices presented, $E_{c c}{ }^{C U R E}$ is calculated using 24 segments (rather than 6) and is high in synchronous contraction with low values indicating dyssynchrony. Comprehensive description of the technique is outside the scope of this paper, but it has been well described elsewhere (17).

\section{Calculation Of Novel “Dyscontractility” Index}

The wide range of indices used to measure dyssynchrony suggests that improvements can be made. We observed that a deeply dyssynchronous heart has significant areas with positive strain values (i.e. focal areas that "balloon" out). We developed a novel index of "dyscontractility" ( $\left.E_{c c}^{\text {MeanMax }}\right)$, calculated as the average of the maximum strains between all segments, with the aim of quantifying this phenomenon (Figure 3). It can be expressed as:

$$
E_{c c}^{M e a n M a x}(s)=\operatorname{mean} \max _{t}\left(E_{C C}(s, t, g)\right),
$$


where $E_{c c}(s, t, g)$ spans the values of strain given $g$ - the number of segment, $t$ - time, and $s$ slice number.

\section{Reproducibility}

Segmentation, slice classification (basal, mid-cavity, apical) and strain calculation were repeated twice for all data sets, once for intra-observer variance assessment, by the same operator with 3 month separation (KW, 5 years of experience) and once for inter-observer variance assessment, by another operator (AD, 2 years of experience).

\section{Statistical Analysis}

Normality of the data was assessed using the D'Agostino-Pearson test. Normally distributed data are presented as mean \pm standard deviation; non-parametric data are presented as median (inter-quartile range). Between-group comparisons were performed using Student T-test (parametric) or Wilcoxon rank sum test (non-parametric). Correlation was measured using Pearson or Spearman correlation coefficient as applicable. Intra- and inter-observer variability and difference between methods are presented as intraclass correlation coefficient with model of absolute agreement among measurements. Areas under receiver operator curves (ROC AUC) were compared using the DeLong method. All statistical tests are 2-tailed, $p$ values $<0.05$ are regarded as significant and presented in bold font. ROC analysis was performed in MedCalc statistical software 14.8.1 (MedCalc, Mariakerke, Belgium). Otherwise statistical analysis was performed in Matlab (Mathworks, USA).

\section{RESULTS}

\section{All Strain Indices Derived From Basal Slice Cine MRI Are Significant Predictors Of CRT Response}

We were able to derive cine strain indices from 48 datasets; 4 cases were lost due to missing short axis cine data. All cine-derived strain indices proved to be statistically significant 
predictors of dyssynchrony when calculated on the basal slice (Table 2), boxplots for all individual cine indices calculated on basal slices are presented in Figure 4). In contrast, the strain indices calculated on mid-cavity and apical slices were not consistently good predictors (Table in Supplementary File).

The novel "dyscontractility" index $\left(E_{c c}{ }^{\text {MeanMax }}\right)$ calculated at the basal level showed the best diagnostic performance numerically $(A U C=0.81)$; this was statistically significant compared to $E_{c c}{ }^{M a x V a r}$ and $E_{c c}{ }^{\operatorname{MaxRVV}}$ (both $p=0.007$ ) with a trend to superiority compared to $E_{c c}{ }^{C U R E}$ and $E_{c c}{ }^{S D I}(p=0.06$ and $p=0.24$ respectively). The AUC for the latter four indices ranged between $0.67-0.76$, without statistical differences between them.

$E_{c c}{ }^{\text {MeanMax }}, E_{c c}{ }^{\text {MaxRVV }}, E_{c c}{ }^{\text {MaxVar }}$ and $E_{c c}{ }^{C U R E}$ calculated from cine imaging all demonstrated reasonable intra- and inter-observer reproducibility $(\mathrm{ICC}>0.9)$, whereas $E_{c c}{ }^{S D I}$ had unacceptably low reproducibility (Table 3).

With an $E_{C C}^{\text {MeanMax }}$ threshold of 4.0 , prediction was made with $83 \%$ specificity, $80 \%$ sensitivity, $81 \%$ accuracy, 20 true positive cases and 4 false positive cases in $\mathrm{N}=48$ population. The Spearman correlation coefficient between the scar percentage (LGE) and $E_{C C}{ }^{\text {MeanMax }}$ was $-0.50(\mathrm{p}<0.01)$. LGE can be a confounding factor between LGE and $E_{C C}$ MeanMax . A logistic regression model between response (dependent variable) and $E_{C C}{ }^{\text {MeanMax }}$ (independent variable) was established with LGE adjusted. The association between response and $E_{C C}{ }^{\text {MeanMax }}$ remained significant $(\mathrm{p}<0.01)$, with risk ratio $(\mathrm{RR})$ of $\mathrm{E}_{\mathrm{CC}}{ }^{\text {MeanMax }}$ equal to 1.641 (CI 1.128-2.387).

\section{Strain Indices Derived From TMRI Were Generally Inferior To Those From Cine MRI, With a Limited Degree Of Correlation}

Strain indices could be calculated from tMRI in 47 datasets; 5 cases were lost due to missing or poor quality tMRI data. In contrast to the predictive ability of cine-derived indices, only $E_{c c}{ }^{M a x R V V}$ and $E_{c c}{ }^{M a x V a r}$ calculated on the basal tMRI slice were significant predictors of 
response to CRT (Table 2), and no tMRI index reached statistical significance on the mid-cavity or apical slices (Table in Supplementary File).

Comparisons between cine and tMRI were possible in the 43 datasets with complete imaging. These comparisons demonstrated only a limited degree of correlation between measures of strain performed across several possible levels of generalization.

There was a moderate correlation between strains derived from either technique across all the segments and time points $(\mathrm{R}=0.54$; Figure 5 left panel). The maximal negative strain values correlated only slightly better $(\mathrm{R}=0.62$; Figure 5 right panel $)$.

The more complex indices derived from strain curves showed very variable degrees of correlation between values calculated from cine and tMRI (Figure 6 top row). Notably, this lack of correlation was associated with generally improved diagnostic performance of indices calculated from cine over those from tMRI (Figure 6 bottom row).

\section{DISCUSSION}

In this study, we propose and validate a novel index of "dyscontractility" as an improved marker of the mechanical consequences of conduction tissue pathology. We show that this index derived on the basal slice compares very favorably to traditional tagged MRI indices of dyssynchrony, with the added advantages of rapid, semi-automated and reliable calculation from ubiquitous cine imaging. Indeed of all indices evaluated, the cine-derived dyscontractility index was numerically the single best predictor of response to cardiac resynchronization therapy in this contemporary heart failure patient cohort.

Our results indicate that dyssynchrony indices calculated in basal slices are better predictors of response to cardiac resynchronization therapy than those calculated in mid-cavity or apical slices. This finding is in accordance with previous reports regarding tMRI (7), and it makes physiological sense, since the basal region contributes more to overall cardiac function and so 
dyskinesia in this region may be relatively more important that dyskinesia in other myocardial segments.

We observed that in more dyssynchronous hearts there are regions with paradoxical positive circumferential strain values, due to either regional variation in the timing of the end-diastolic phase within the cardiac cycle, or the presence of dyskinetic regions of myocardium. Based on this simple observation, we proposed a novel "dyscontractility" index $\left(E_{c c}{ }^{\text {MeanMax }}\right)$. Henceforth, this is referred to as "cine dyscontractility index" or " $C D I$ ". The interpretation of $C D I$ is very intuitive - it is zero if the whole myocardium contracts with reference to the initial (end diastolic) state, and it increases if either the end diastolic phase is different for some parts of the myocardium or if there is a dyskinetic region of myocardium. Of the established indices that we also evaluated, those indices measuring spatial variance of strain $\left(E_{c c}{ }^{M a x R V}\right.$ and $\left.E_{c c}{ }^{M a x V a r}\right)$ came closest to matching the performance of $C D I$ and were reasonably good predictors of CRT response. The index based on temporal variance of $\operatorname{strain}\left(E_{c c}{ }^{S D I}\right)$ showed reasonable predictive value, but was limited by very poor intra-observer and inter-observer reproducibility. In highly dyssynchronous hearts $E_{c c}$ is positive through almost the entire cardiac cycle; in such cases the $E_{c c}$ minimum is either at the beginning or at the end of the cycle and small changes in segment distribution may shift it considerably, leading to large variations in calculated $E_{c c}{ }^{S D I}$.

In our study, cine-based indices expressed more numerous statistically significant associations with response to CRT than equivalently processed tMRI data, which calls for some discussion on the differences between the underlying imaging techniques. Cine MRI is acquired using bSSFP sequences, and generally has high SNR between tissues, but the image contrast within the cardiac muscle is low. This implies that tracking anatomy and tissue borders is easy, but motion or strain tracking within the muscle itself is challenging. The latter can be addressed by dedicated tMRI, as the artificially induced tags allow tracking of both motion and internal distortion within the myocardial tissue, overcoming the lack of intrinsic contrast. 
However, this comes at the price of both reduced spatial resolution $(\sim 5-10 \mathrm{~mm}$ inter-tag-spacing), resulting in blurring of much detail of cardiac anatomy, and also typically lower temporal resolution. The tagged grid appearance decays visibly over time, which worsens SNR and may disproportionately affect indices that rely on tag detection late into the cardiac cycle.

Thus, expectedly and in line with prior literature (21), both approaches provide linked but certainly non-identical measures of strain. In our cohort, results were modestly correlated between the two methods on both a segmento-temporal and minimal strain in segment basis. There were two dyssynchrony indices that showed prognostic value in both cine and tMRI ( $E_{c c}{ }^{M a x V a r}$ and $E_{c c}{ }^{\operatorname{MaxRVV}}$ ), which correlated well between methods. On the other hand, this was not the case with CDI, which showed excellent diagnostic performance but only modest correlation with its tMRI-derived counterpart. Mechanistically, this difference may firstly reflect the greater sensitivity of the cine outline to longitudinal descent of the basal segments in systole - i.e. through-slice motion. For tMRI, through-slice motion will shift tag lines from outside the imaging plane, but the inter-tag distances (and calculation of "tissue" strain) are expected to remain unaffected. In contrast, the cine imaging outline (and hence cine-derived calculation of "myocardial outline " stretch) are expected to be affected by through-slice motion; this additional sensitivity to detection of dysschronous long-axis motion compared to tMRI could potentially be relevant to the observed differences in performance. Secondly, the difference may also be exacerbated by the fact that the CDI calculation highlights the presence and extent of "dyscontractility" (i.e. small positive strains) at any cardiac phase, including very late timings where tMRI tags may have largely dissipated. The presence of counterintuitive myocardial "ballooning" appears to be an essential underpinning factor for clinical improvement following CRT. Conversely, CDI caps the effect of any differences between the negative strain curves, which are the recognised strength of widely accepted early/systolic 
phase maximum strain tMRI imaging. In this case, the negative strain range appears to carry less information on CRT outcomes, and may potentially introduce noise into other indices, thus explaining their weaker diagnostic performance compared to CDI. These features of CDI are also consistent with the observed excellent intra-observer and inter-observer reproducibility. Whilst we believe these are all plausible explanations for our findings, we have not assessed these potential mechanisms directly and the discussion remains speculative pending further investigation, perhaps using 3D tissue motion modelling approaches.

Irrespective of the underlying mechanistic explanation for our findings, there are a number of reasons for which we believe that cine-MRI derived strain has a number of advantages over tMRI for routine clinical use. Firstly, $C D I$ was numerically the single best predictor of response to resynchronisation therapy, and compared well with the best tMRI index $\left(E_{c c}{ }^{M a x R V V}\right)$. Secondly, cine images are ubiquitously acquired and this obviation of the need for additional breath-holds offers clear clinical benefit. This is particularly relevant to the assessment of patients with cardiac failure who often find it difficult to perform the relatively long breath-holds, generally necessary for tMRI acquisitions (22). Finally, $C D I$ is very simple to process, with operator input limited to standard placement of endocardial and epicardial contours and marking of the right ventricular insertion point in the end diastolic phase. It can also be rapidly derived, with our implementation taking only about 1 minute to calculate the displacement field in a series of 25 images from one slice on a consumer level PC $(2,3 \mathrm{GHz}$ Intel Core i7, 8GB RAM). Our results suggest that calculation of $C D I$ on a single basal slice is sufficient to derive useful prognostic information. Nevertheless, if processing of a whole short axis cine stack is desired, this currently takes around 10-15 minutes, and would be further reduced with code optimisations and application of heavily parallelized GPU processing.

Our results indicate that $C D I$ can better predict response to CRT than conventional tMRI-derived indices, and may therefore represent an improved imaging marker of mechanical 
dyssynchrony. If this is confirmed in further studies, there is potential for significant clinical implications. The ubiquitous nature of cine imaging means that normal ranges for $C D I$ could be relatively rapidly derived and used to define objective thresholds for dyskinesia. The next step would be a systematic evaluation of a large number of patients with heart failure, both with and without QRS broadening on ECG. The additive benefit of the MRI dyssynchrony data above and beyond QRS duration and LV ejection fraction would then be clearly defined, and in the first instance these data could guide decisions regarding CRT therapy in cases which are borderline based on current clinical guidelines. Ultimately, MRI assessment might accurately identify dyssynchrony in heart failure patients with narrow QRS complexes. Previous experience of CRT implantation based on echocardiographic dyssynchrony indices in this patient group demonstrated no benefit and possible harm (23). However, the base of the heart is much better visualised using MRI compared to echocardiography, and improved quantification of dyssynchrony in this region using $C D I$ could potentially lead to better outcomes.

This study represents the first step in validation of $C D I$ in a relatively small number of study subjects. We only had access to MRI data prior to CRT implantation - future studies that recruit patients undergoing MRI-conditional device implantation may be able to define changes in dyssynchrony and reverse remodelling of the left ventricle after device implantation. Similarly, the impact of additional information about left ventricular scar location on late gadolinium imaging (6) and/or the left ventricular electrogram timings (8) should be assessed in future work. It is also possible that the index could be improved by further modifications; for example, some studies show that limiting an index calculation spatially to the septal region (24) or temporally to the ejection phase (25) improve predictive value. Additionally, advancements in SSFP tagging and CSPAMM techniques offering different insights into heart motion (22) also warrant further investigation. 
In conclusion, cine dyscontractility index $(C D I)$ is a novel marker of traditional dyssynchrony and conduction tissue pathology, compares favourably to established indices for the prediction of response to CRT therapy, and can be rapidly derived from routinely collected cine imaging data. Further prospective studies are needed to validate $C D I$ as a marker of dyssynchrony, and assess its potential clinical utility.

\section{REFERENCES}

1. Bui AL, Horwich TB, Fonarow GC. Epidemiology and risk profile of heart failure. Nat Rev Cardiol. 2011 Jan;8(1):30-41.

2. Leclercq C, Kass DA. Retiming the failing heart: principles and current clinical status of cardiac resynchronization. J Am Coll Cardiol. 2002 Jan 16;39(2):194-201.

3. McAlister FA, Ezekowitz J, Hooton N, Vandermeer B, Spooner C, Dryden DM, et al. Cardiac resynchronization therapy for patients with left ventricular systolic dysfunction: a systematic review. JAMA. 2007 Jun 13;297(22):2502-14.

4. Ypenburg C, van Bommel RJ, Borleffs CJW, Bleeker GB, Boersma E, Schalij MJ, et al. Long-Term Prognosis After Cardiac Resynchronization Therapy Is Related to the Extent of Left Ventricular Reverse Remodeling at Midterm Follow-Up. J Am Coll Cardiol. 2009 Feb;53(6):483-90.

5. Chung ES, Leon AR, Tavazzi L, Sun J-P, Nihoyannopoulos P, Merlino J, et al. Results of the Predictors of Response to CRT (PROSPECT) Trial. Circulation. 2008 May 20;117(20):2608-16.

6. Bilchick KC, Dimaano V, Wu KC, Helm RH, Weiss RG, Lima JA, et al. Cardiac Magnetic Resonance Assessment of Dyssynchrony and Myocardial Scar Predicts Function Class Improvement Following Cardiac Resynchronization Therapy. JACC Cardiovasc Imaging. 2008 Sep;1(5):561-8.

7. Petryka J, Miśko J, Przybylski A, Śpiewak M, Małek ŁA, Werys K, et al. Magnetic resonance imaging assessment of intraventricular dyssynchrony and delayed enhancement as predictors of response to cardiac resynchronization therapy in patients with heart failure of ischaemic and non-ischaemic etiologies. Eur J Radiol. 2012 Oct;81(10):2639-47.

8. Bilchick KC, Kuruvilla S, Hamirani YS, Ramachandran R, Clarke SA, Parker KM, et al. Impact of Mechanical Activation, Scar, and Electrical Timing on Cardiac Resynchronization Therapy Response and Clinical Outcomes. J Am Coll Cardiol. 2014 Apr;63(16):1657-66.

9. Riffel JH, Andre F, Maertens M, Rost F, Keller MG, Giusca S, et al. Fast assessment of long axis strain with standard cardiovascular magnetic resonance: a validation study of a novel parameter with reference values. J Cardiovasc Magn Reson. 2015 Aug 8;17(1):69. 
10. Werys K, Błaszczyk Ł, Kubik A, Marczak M, Bogorodzki P. Displacement field calculation from CINE MRI using non-rigid image registration. In: The 8th IEEE International Conference on Intelligent Data Acquisition and Advanced Computing Systems: Technology and Applications. Warsaw; 2015.

11. Rueckert D, Sonoda LI, Hayes C, Hill DL, Leach MO, Hawkes DJ. Nonrigid registration using free-form deformations: application to breast MR images. IEEE Trans Med Imaging. 1999 Aug;18(8):712-21.

12. Ledesma-Carbayo MJ, Mahía-Casado P, Santos A, Pérez-David E, García-Fernández MA, Desco M. Cardiac motion analysis from ultrasound sequences using nonrigid registration: Validation against Doppler tissue velocity. Ultrasound Med Biol. 2006 Apr;32(4):483-90.

13. Cerqueira MD. Standardized Myocardial Segmentation and Nomenclature for Tomographic Imaging of the Heart: A Statement for Healthcare Professionals From the Cardiac Imaging Committee of the Council on Clinical Cardiology of the American Heart Association. Circulation. 2002 Jan 29;105(4):539-42.

14. Arts T, Prinzen FW, Delhaas T, Milles JR, Rossi AC, Clarysse P. Mapping Displacement and Deformation of the Heart With Local Sine-Wave Modeling. IEEE Trans Med Imaging. 2010 May;29(5):1114-23.

15. Helm PA. Evidence of Structural Remodeling in the Dyssynchronous Failing Heart. Circ Res. 2005 Dec 8;98(1):125-32.

16. Helm RH. Cardiac Dyssynchrony Analysis Using Circumferential Versus Longitudinal Strain: Implications for Assessing Cardiac Resynchronization. Circulation. 2005 May 31;111(21):2760-7.

17. Sohal M, Duckett SG, Zhuang X, Shi W, Ginks M, Shetty A, et al. A prospective evaluation of cardiovascular magnetic resonance measures of dyssynchrony in the prediction of response to cardiac resynchronization therapy. J Cardiovasc Magn Reson. 2014 Aug 1;16(1):58.

18. Leclercq C. Systolic Improvement and Mechanical Resynchronization Does Not Require Electrical Synchrony in the Dilated Failing Heart With Left Bundle-Branch Block. Circulation. 2002 Oct 1;106(14):1760-3.

19. Budge LP, Helms AS, Salerno M, Kramer CM, Epstein FH, Bilchick KC. MR Cine DENSE Dyssynchrony Parameters for the Evaluation of Heart Failure. JACC Cardiovasc Imaging. 2012 Aug;5(8):789-97.

20. El Ghannudi S, Germain P, Jeung M-Y, Breton E, Croisille P, Durand E, et al. Quantification of left ventricular dyssynchrony in patients with systolic dysfunction: A comparison of circumferential strain MR-tagging metrics: Quantification of LV Dyssynchrony. J Magn Reson Imaging. 2014 Nov;40(5):1238-46.

21. Wu L, Germans T, Güçlü A, Heymans MW, Allaart CP, Rossum AC van. Feature tracking compared with tissue tagging measurements of segmental strain by cardiovascular magnetic resonance. J Cardiovasc Magn Reson. 2014 Jan 22;16(1):10. 
22. Ibrahim ESH. Myocardial tagging by Cardiovascular Magnetic Resonance: evolution of techniques-pulse sequences, analysis algorithms, and applications. J Cardiovasc Magn Reson. 2011;13(1):1-40.

23. Ruschitzka F, Abraham WT, Singh JP, Bax JJ, Borer JS, Brugada J, et al. Cardiac-resynchronization therapy in heart failure with a narrow QRS complex. N Engl J Med. 2013 Oct 10;369(15):1395-405.

24. De Boeck BWL, Teske AJ, Meine M, Leenders GE, Cramer MJ, Prinzen FW, et al. Septal rebound stretch reflects the functional substrate to cardiac resynchronization therapy and predicts volumetric and neurohormonal response. Eur J Heart Fail. 2009 Sep;11(9):863-71.

25. Kirn B, Jansen A, Bracke F, van Gelder B, Arts T, Prinzen FW. Mechanical discoordination rather than dyssynchrony predicts reverse remodeling upon cardiac resynchronization. Am J Physiol Heart Circ Physiol. 2008 Aug;295(2):H640-6. 
Table 1 Baseline clinical characteristics and MRI parameters in the study group.

\begin{tabular}{|c|c|c|c|c|}
\hline Variable & Study group & Responders & Non-responders & p value \\
\hline & $\mathrm{N}=52$ & $\overline{\mathrm{N}}=26$ & $\mathrm{~N}=26$ & \\
\hline Age (years) & $60.3 \pm 13.0$ & $59.6 \pm 12.9$ & $61.0 \pm 13.3$ & 0.69 \\
\hline $\operatorname{Sex}(\%$ men $)$ & $44(84.6 \%)$ & $20(76.9 \%)$ & $24(92.3 \%)$ & 0.13 \\
\hline BMI (kg/m2) & $28.1 \pm 4.4$ & $27.3 \pm 3.7$ & $29.0 \pm 5.0$ & 0.20 \\
\hline \multirow{3}{*}{ NYHA class II-IV } & II - $14(27 \%)$ & II - $5(19 \%)$ & II - $9(36 \%)$ & \\
\hline & III - $31(59 \%)$ & III - $17(66 \%)$ & III - $13(52 \%)$ & 0.32 \\
\hline & IV-7(14\%) & IV $-4(15 \%)$ & IV - $3(12 \%)$ & \\
\hline QRS duration (ms) & $160.0 \pm 24.4$ & $165.0 \pm 21.8$ & $153.9 \pm 26.6$ & \\
\hline $\begin{array}{l}\text { Creatinine levels } \\
(\mathrm{mg} / \mathrm{ml})\end{array}$ & $101.6 \pm 27.5$ & $98.3 \pm 25.9$ & $105.1 \pm 29.2$ & 0.39 \\
\hline $\begin{array}{l}\text { NT-proBNP levels } \\
(\mathrm{pg} / \mathrm{ml})\end{array}$ & $1517(575-3457)$ & $1760(506-4302)$ & $1367(693-3397)$ & 0.89 \\
\hline LVEF by echo $(\%)$ & $23.9 \pm 6.6$ & $23.9 \pm 7.4$ & $23.9 \pm 5.8$ & 0.99 \\
\hline $\begin{array}{l}\mathrm{VO} 2 \mathrm{max} \\
(\mathrm{ml} / \mathrm{kg} / \mathrm{min})\end{array}$ & $12.9 \pm 3.6$ & $12.7 \pm 3.3$ & $13.3 \pm 4.2$ & 0.71 \\
\hline LGE (\%) & $13.4(0-25.7)$ & $7.7(0-13.5)$ & $19.0(0-31.9)$ & 0.013 \\
\hline
\end{tabular}

BMI - Body Mass Index, NYHA - New York Heart Association, NT-proBNP - N-terminal of the prohormone brain natriuretic peptide, LVEF - left ventricular ejection fraction, VO2 - peak oxygen consumption, LGE - late gadolinium enhancement. 
Table 2 Dyssynchrony indices that are significant CRT response predictors. P values relate to the between group comparison (responders vs. non-responders) and AUC relates to the ability of the index to predict response to CRT. The complete results for all indices at each cardiac level are provided in the Supplementary File.

\begin{tabular}{|c|c|c|c|c|c|}
\hline & Level & Responders & Non-responders & p value & AUC \\
\hline Cine & & $\mathrm{N}=23$ & $\mathrm{~N}=25$ & & \\
\hline \multirow[t]{2}{*}{$E_{c c}^{M e a n M a x}$} & Base & $5.95(4.23-7.48)$ & $2.67(1.30-3.66)$ & $<0.001$ & 0.81 \\
\hline & Mid & $4.63(3.22-7.02)$ & $3.01(1.17-4.56)$ & 0.014 & 0.70 \\
\hline$E_{c c}^{S D I}$ & Base & $19.9(17.1-27.0)$ & $13.1(10.7-20.7)$ & 0.002 & 0.76 \\
\hline \multirow[t]{2}{*}{$E_{c c}^{M a x R V V}$} & Base & $53.4(35.9-65.3)$ & $44.3(32.2-53.0)$ & 0.043 & 0.67 \\
\hline & Mid & $39.0(31.9-56.4)$ & $30.9(23.9-41.7)$ & 0.024 & 0.69 \\
\hline \multirow[t]{2}{*}{$E_{c c}^{M a x V a r}$} & Base & $243.8(151.0-375.0)$ & $155.1(83.1-218.7)$ & 0.013 & 0.71 \\
\hline & Mid & $156.5(87.1-288.6)$ & $86.8(64.7-132.3)$ & 0.027 & 0.68 \\
\hline$E_{c c}{ }^{C U R E}$ & Base & $64.8(49.0-72.7)$ & $75.9(65.9-84.7)$ & 0.014 & 0.71 \\
\hline tMRI & & $(\mathrm{N}=24)$ & $(\mathrm{N}=23)$ & & \\
\hline$E_{c c}{ }^{\operatorname{Max} R V V}$ & Base & $35.0(26.6-38.8)$ & $27.9(18.8-33.7)$ & 0.013 & 0.71 \\
\hline$E_{c c}^{M a x V a r}$ & Base & $94.7(70.1-118.1)$ & $69.8(48.1-93.9)$ & 0.042 & 0.67 \\
\hline
\end{tabular}

$\boldsymbol{E}_{\boldsymbol{c c}}$ MeanMax - average of the maximum strains dyssynchrony index, $\boldsymbol{E}_{\boldsymbol{c}} \boldsymbol{S D I}$ - strain dyssynchrony index, $\boldsymbol{E}_{c c} \operatorname{MaxRVV}$ - maximum regional variance vector dyssynchrony

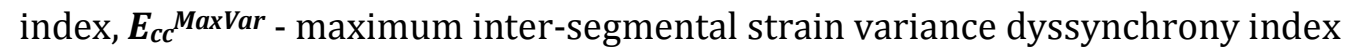
$\boldsymbol{E}_{c c}$ CURE - circumferential uniformity ratio estimate dyssynchrony index, AUC - area under Receiver operating characteristic curve. Statistically significant $\mathrm{p}$ values are in bold. 
Table 3 Intra-observer and inter-observer reproducibility for cine-derived indices in all slices. Variability reflects both slice classification and segmentation.

\begin{tabular}{|c|c|c|c|c|c|c|c|}
\hline \multicolumn{3}{|c|}{ Intra-observer } & \multicolumn{5}{|c|}{ Inter-observer } \\
\hline Index & Obs. 1 & Obs. 1 repeat & $\overline{\mathrm{D} \text { Diff } \pm \mathrm{SD}}$ & $\overline{\mathrm{ICC}}$ & Obs. 2 & $\overline{\mathrm{D}} \mathrm{Diff} \pm \mathrm{SD}$ & $\overline{\mathrm{ICC}}$ \\
\hline$E_{c c}^{\text {MeanMax }}$ & $4.36(2.36-6.12)$ & $4.21(2.35-5.96)$ & $0.02 \pm 1.09$ & 0.90 & $4.36(2.53-6.34)$ & $0.17 \pm 0.91$ & 0.93 \\
\hline$E_{c c}^{S D I}$ & $20.2(14.9-26.7)$ & $20.7(15.7-26.6)$ & $0.40 \pm 8.62$ & 0.57 & $19.5(13.5-23.8)$ & $1.06 \pm 9.13$ & 0.42 \\
\hline$E_{c c}^{M a x R V V}$ & $38.4(31.9-48.5)$ & $36.7(31.8-45.9)$ & $0.73 \pm 3.82$ & 0.96 & $40.2(33.1-49.3)$ & $2.57 \pm 5.11$ & 0.91 \\
\hline$E_{c c}^{M a x V a r}$ & $130(93-225)$ & $129(88-192)$ & $6.4 \pm 44.1$ & 0.92 & $143(107-243)$ & $25.1 \pm 49.8$ & 0.90 \\
\hline$E_{c c}^{C U R E}$ & $67.4(50.5-77.5)$ & $67.9(53.1-77.2)$ & $0.86 \pm 3.83$ & 0.97 & $66.8(52.5-76.7)$ & $0.31 \pm 7.15$ & 0.91 \\
\hline
\end{tabular}

$\boldsymbol{E}_{c c}$ MeanMax - average of the maximum strains dyssynchrony index, $\boldsymbol{E}_{c c} \boldsymbol{S D I}$ - strain dyssynchrony index, $\boldsymbol{E}_{c c^{M a x} \boldsymbol{M V} \boldsymbol{V}}$ - maximum regional variance vector dyssynchrony index, $\boldsymbol{E}_{\boldsymbol{c c}} \boldsymbol{c}^{\text {MaxVar }}$ - maximum inter-segmental strain variance dyssynchrony index $\boldsymbol{E}_{c c} C U R E$ - circumferential uniformity ratio estimate dyssynchrony index, ICC intraclass correlation coefficient. 
Figure 1 Representative circumferential strain curve plots and circumferential strain maps. Example maps are provided from basal slices of (top) a visibly dyssynchronous CRT-responder and (bottom) a non-responder with relatively good baseline synchrony. Values in the plot are calculated as median circumferential strain value in each of the 6 segments. In the patient with dyssynchrony, a region of positive circumferential strain is clearly visible both on the curves (pink arrow) and on the maps (red region within myocardial outline 7-11 o'clock, pink arrow).

Figure 2 A - Representative examples of calculation of $E_{c c}{ }^{S D I}$ from circumferential strain curve plots. The times to minimum strain value (ttmin) were calculated (presented as arrows), and the standard deviation of those times was taken. In the dyskinetic heart, some segments (such as the inferoseptum shown in green in left panel) have positive $E_{c c}$ through the entire cardiac cycle. This means that tmin occurs close to either the beginning or the end of the cycle and results in high variability from other segments and hence high $E_{c c}{ }^{S D I}$. In contrast, in a synchronous left ventricle (right panel), ttmin values are similar in all segments, resulting in low $E_{c c}{ }^{S D I}$. B - Graphical illustrations of $\boldsymbol{E}_{c c}{ }^{M a x R V V}$ calculation. Radial vectors of segmental strain are evenly distributed on the border of a circle (top panels, black arrows), and are summed to summarize the overall shape strain (regional variance vector - RVV, thick green arrow). $E_{c c}{ }^{M a x R V V}$ is defined as the maximum length of the RVV, shown as "max" on the graph of RVV evolution over time (bottom panels). In mechanical dyssynchrony (left panels), the opposite vectors sum to generate a high $E_{c c}{ }^{M a x R V V}$ value. In contrast, in uniform and synchronous myocardial contraction (right panels), the opposite vectors cancel each other out and $E_{c c}{ }^{M a x R V V}$ is low. C - Representative examples of inter-segmental strain variance through the cardiac cycle. Discrepancies between strain curves are high in mechanical dyssynchrony (left panel), especially in the systolic phase ( $E_{c c}{ }^{\text {MaxVar }}$ high). In a synchronous left ventricle (right panel), variance is low through the entire cardiac cycle ( $E_{c c}{ }^{\text {MaxVar }}$ low). 
Figure 3 Representative examples of calculation of the novel dyscontractility index $\left(E_{c c}{ }^{\text {MeanMax }}\right)$ from circumferential strain curve plots. In a case of mechanical dyssynchrony (left panel), there is a cardiac region with positive circumferential strain, resulting in high $E_{c c}{ }^{\text {MeanMax }}$. In contrast in a synchronous left ventricle (right panel), circumferential strain is negative during the whole cardiac cycle resulting in low $E_{c c}^{\text {MeanMax }}$.

Figure 4 Boxplots for the cine dyssynchrony indices calculated on the basal slices. All comparisons between CRT non-responders (NonResp) and responders (Resp) were statistically significant $($ all $\mathrm{p}<0.05)$. Note that in contrast to other indices, a low value of $E_{c c}{ }^{C U R E}$ reflects dyssynchrony.

Figure 5 Comparison of results between cine MRI and tMRI. Points in spatial/temporal $E_{c c}$ scatter plot (left panel) represent every point on the strain curves shown in Figure 1 across all segments, all slices and all individuals. Points in $\min E_{c c}$ scatter plot (right panel) represent the minimal value amongst all curves in each slice in all individuals.

Figure 6 Comparison of individual strain indices derived from cine and tMRI. Correlation between individual values (top row) and diagnostic performance using ROC analysis (bottom row) are shown. Note the moderate levels of correlation and disparities in diagnostic performance. In particular cine $E_{c c}^{M e a n M a x}$ and $E_{c c}{ }^{S D I}$ indices are poorly correlated with their tMRI counterparts, resulting in a clear diagnostic advantage to the cine techniques $(p=0.005$ and $\mathrm{p}=0.044$, respectively). 

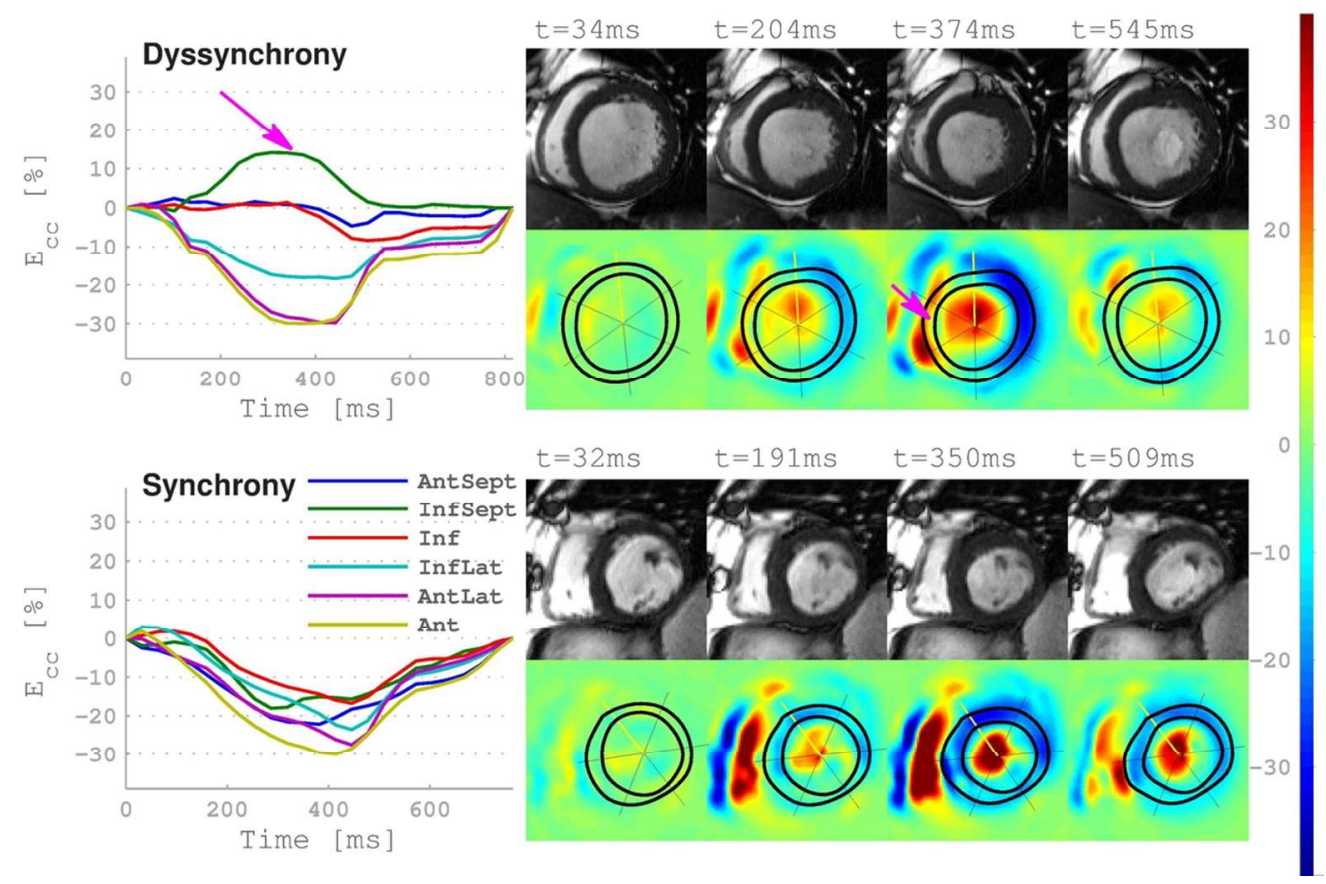

Representative circumferential strain curve plots and circumferential strain maps. Example maps are provided from basal slices of (top) a visibly dyssynchronous CRT-responder and (bottom) a non-responder with relatively good baseline synchrony. Values in the plot are calculated as median circumferential strain value in each of the 6 segments. In the patient with dyssynchrony, a region of positive circumferential strain is clearly visible both on the curves (pink arrow) and on the maps (red region within myocardial outline 7-11 o'clock, pink arrow).

$102 \times 68 \mathrm{~mm}(300 \times 300 \mathrm{DPI})$ 

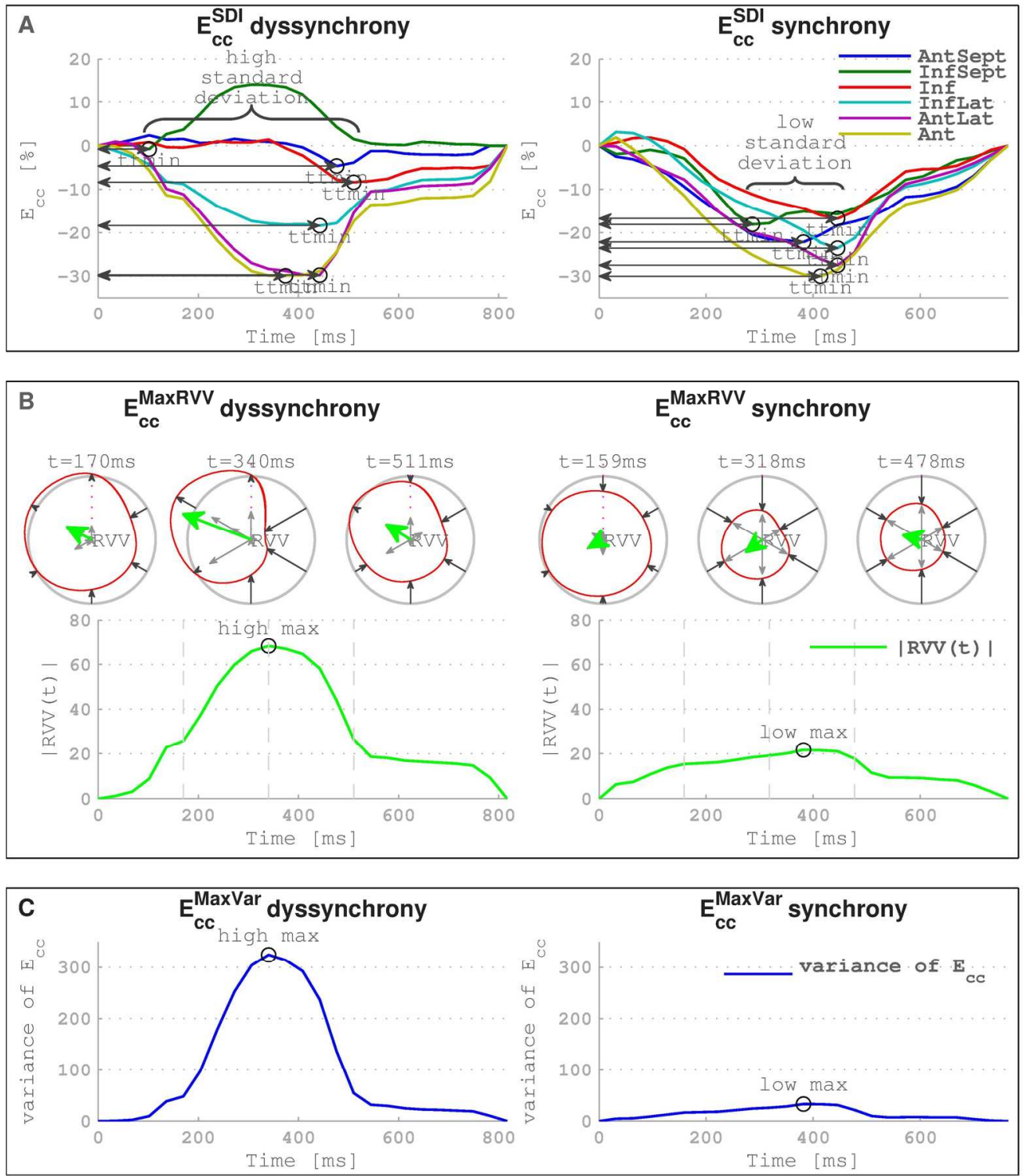

A - Representative examples of calculation of EccSDI from circumferential strain curve plots. The times to minimum strain value (ttmin) were calculated (presented as arrows), and the standard deviation of those times was taken. In the dyskinetic heart, some segments (such as the inferoseptum shown in green in left panel) have positive Ecc through the entire cardiac cycle. This means that ttmin occurs close to either the beginning or the end of the cycle and results in high variability from other segments and hence high EccSDI. In contrast, in a synchronous left ventricle (right panel), ttmin values are similar in all segments, resulting in low EccSDI. B - Graphical illustrations of EccMaxRVV calculation. Radial vectors of segmental strain are evenly distributed on the border of a circle (top panels, black arrows), and are summed to summarize the overall shape strain (regional variance vector - RVV, thick green arrow). EccMaxRVV is defined as the maximum length of the RVV, shown as "max" on the graph of RVV evolution over time (bottom panels). In mechanical dyssynchrony (left panels), the opposite vectors sum to generate a high EccMaxRVV value. In contrast, in uniform and synchronous myocardial contraction (right panels), the opposite vectors cancel each other out and EccMaxRVV is low. C - Representative examples of inter-segmental strain variance through the cardiac cycle. Discrepancies between strain curves are high in mechanical dyssynchrony (left panel), especially in the systolic phase (EccMaxVar high). In a synchronous left ventricle (right panel), variance is 
low through the entire cardiac cycle (EccMaxVar low). $176 \times 204 \mathrm{~mm}(300 \times 300$ DPI) 

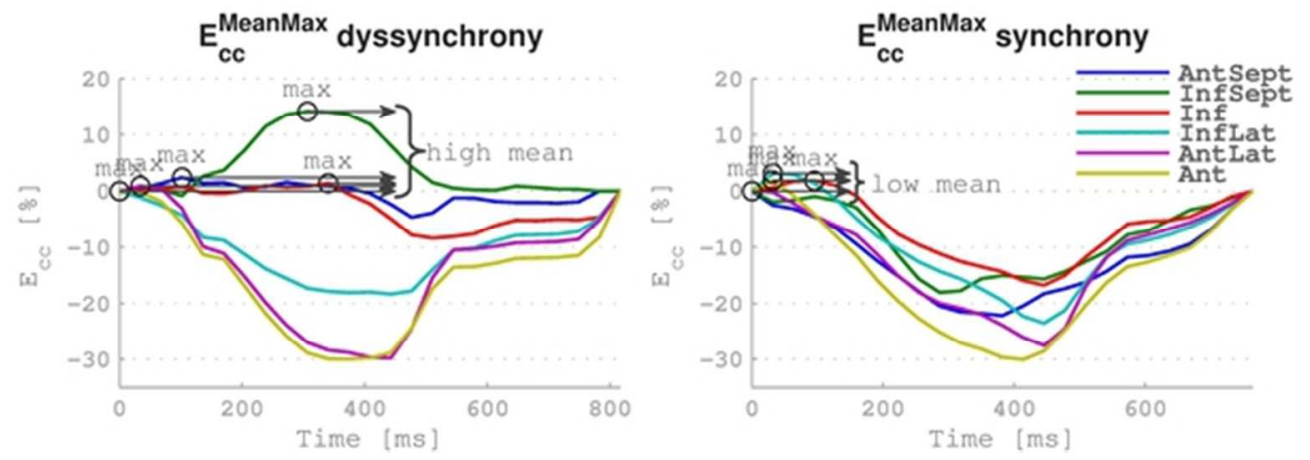

Representative examples of calculation of the novel dyscontractility index (EccMeanMax) from circumferential strain curve plots. In a case of mechanical dyssynchrony (left panel), there is a cardiac region with positive circumferential strain, resulting in high EccMeanMax. In contrast in a synchronous left ventricle (right panel), circumferential strain is negative during the whole cardiac cycle resulting in low EccMeanMax. $51 \times 17 \mathrm{~mm}(300 \times 300$ DPI $)$ 


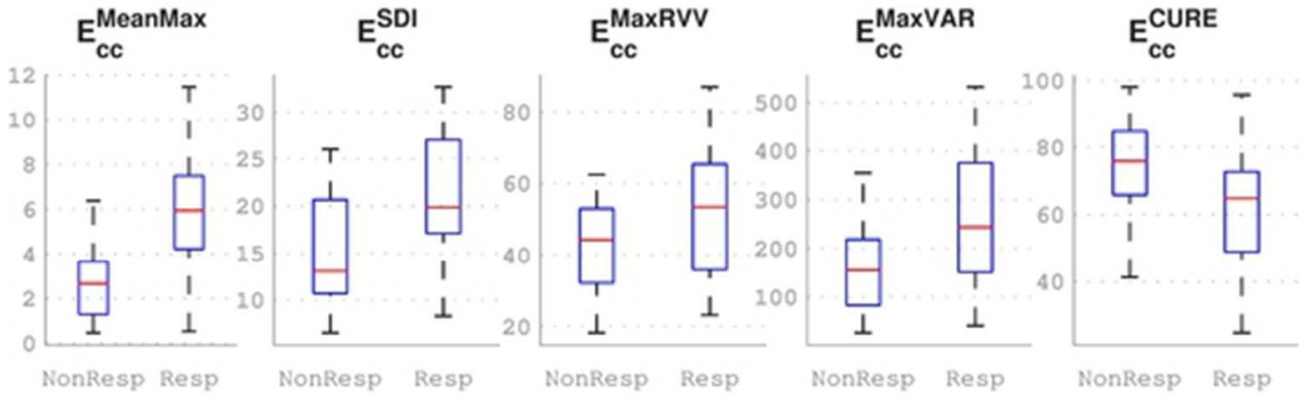

Boxplots for the cine dyssynchrony indices calculated on the basal slices. All comparisons between CRT nonresponders (NonResp) and responders (Resp) were statistically significant (all p < 0.05). Note that in contrast to other indices, a low value of EccCURE reflects dyssynchrony. $47 \times 14 \mathrm{~mm}(300 \times 300$ DPI) 

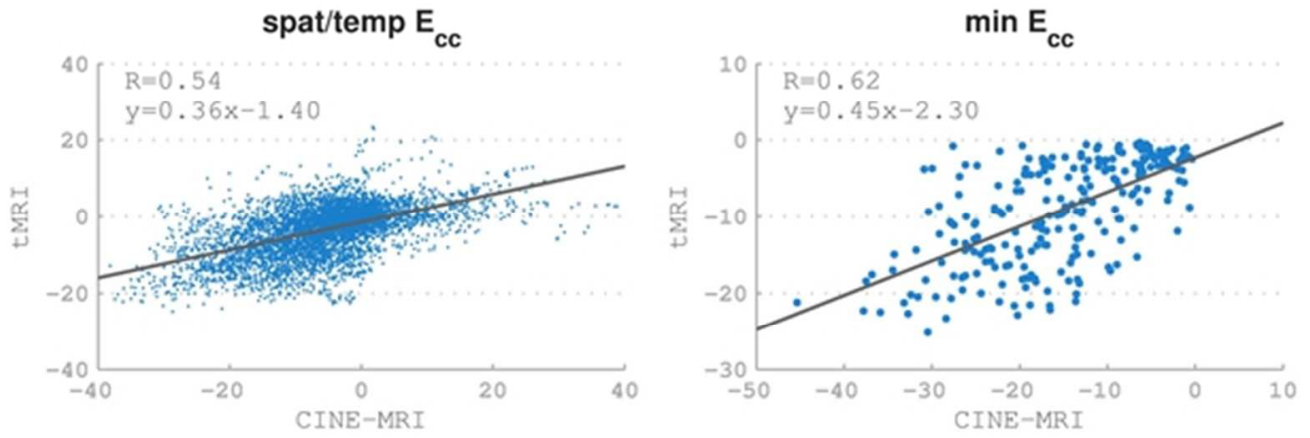

Comparison of results between cine MRI and tMRI. Points in spatial/temporal Ecc scatter plot (left panel) represent every point on the strain curves shown in Figure 1 across all segments, all slices and all individuals. Points in minEcc scatter plot (right panel) represent the minimal value amongst all curves in each slice in all individuals. $51 \times 17 \mathrm{~mm}(300 \times 300 \mathrm{DPI})$ 

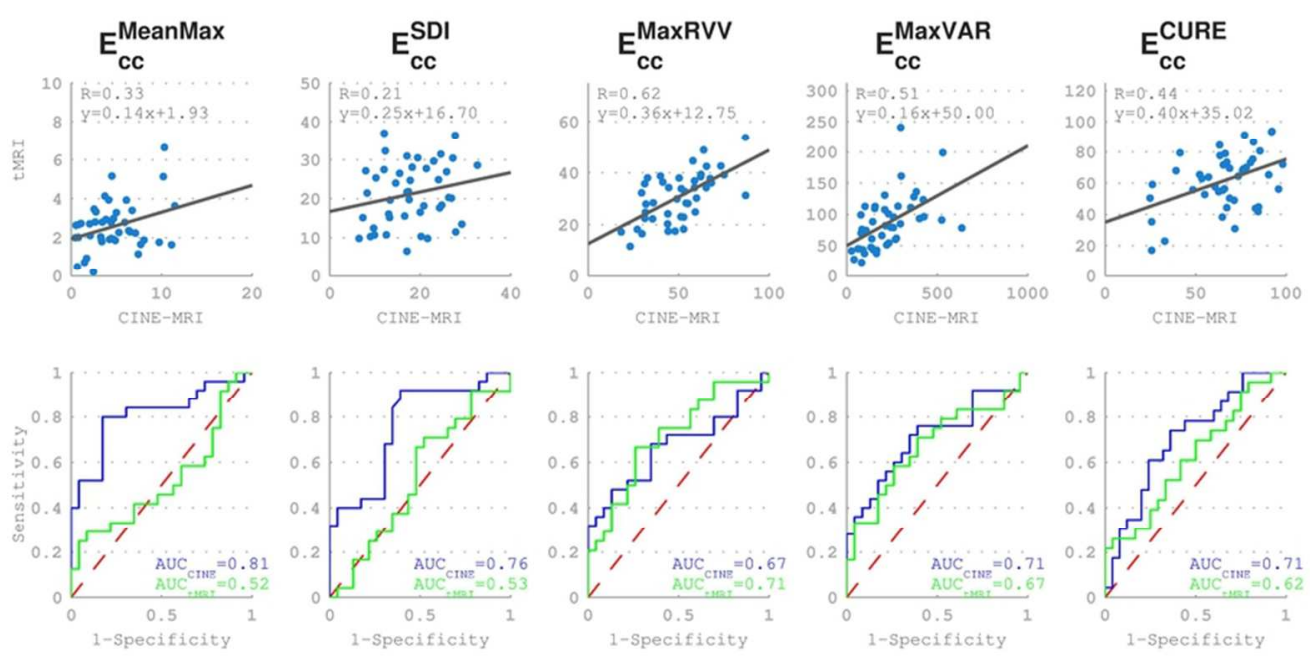

Comparison of individual strain indices derived from cine and tMRI. Correlation between individual values (top row) and diagnostic performance using ROC analysis (bottom row) are shown. Note the moderate levels of correlation and disparities in diagnostic performance. In particular cine EccMeanMax and EccSDI indices are poorly correlated with their tMRI counterparts, resulting in a clear diagnostic advantage to the cine techniques $(p=0.005$ and $p=0.044$, respectively). 